\begin{tabular}{|c|c|c|}
\hline 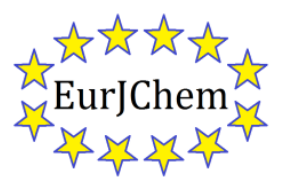 & $\begin{array}{c}\text { European Journal of Chemistry } \\
\text { Journal homepage: } \underline{\text { www.eurjchem.com }}\end{array}$ & 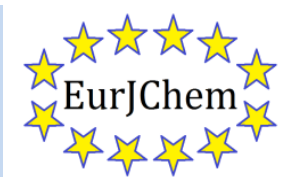 \\
\hline
\end{tabular}

\title{
Synthesis of octahydroquinazolinone derivatives using silica sulfuric acid as an efficient catalyst
}

\author{
Akbar Mobinikhaledia,*, Naser Foroughifara,b and Homila Khodaeia \\ a Department of Chemistry, Arak University, Arak, IR-38156-879, Iran \\ b Faculty of Chemistry, Islamic Azad University, North Tehran Branch, IR-1491367711, Iran \\ *Corresponding author at: Department of Chemistry, Arak University, Arak, IR-38156-879, Iran. Tel.: +98.861.2774031; fax: +98.861.2774031. \\ E-mail address: akbar mobini@yahoo.com (A. Mobinikhaledi).
}

\section{ARTICLE INFORMATION}

\section{Received: 14 May 2010}

Received in revised form: 21 June 2010

Accepted: 14 August 2010

Online: 31 December 2010

\section{KEYWORDS}

Quinazolinone

Silica sulfuric acid

Dimedone

Biginelli reaction

\section{ABSTRACT}

Octahydroquinazolinone derivatives were synthesized in moderate to high yields via one-pot three component Biginelli reaction of dimedone, urea or thiourea and corresponding aromatic aldehydes in the presence of silica sulfuric acid as an efficient catalyst, in ethanol under reflux conditions. This protocol offers several advantages including good yields of products and easy experimental work-up procedure.

\section{Introduction}

Over the years, research interest for the synthesis of dihydro pyrimidinones and their derivatives including octahydroquinazolinone using multicomponents reactions has surged rapidly, owing to the diverse pharmacological properties associated with the pyrimidine core [1,2]. Octahydroquinazolinone derivatives have received considerable attention within recent years due to their attractive pharmacological profiles [2-5]. Several methods have been reported for the synthesis of quinazolinone derivatives $[6,7]$. These methods usually involve the reaction of an aldehyde with thionyl chloride and pyridine, which is followed in reaction with 2-aminobenzylamine in different organic solvents. However, most of these methods have limitations including harsh reaction conditions, low yields, use of expensive and hazardous chemicals, and tedious workup procedure. The most general method for the preparation of octahydroquinazolinones involves the one-pot Biginelli condensation reaction of dimedone, aromatic aldehydes and urea/or thiourea in the presence of a Lewis or mineral acids $[5,8]$. Although various Lewis acid catalysts have been used in the extension of the Biginelli reaction, they are harmful, expensive and difficult to handle [9-13]. Consequently, the introduction of new methods and /or further work on technical improvements to overcome these limitations is still needed. Recently, the use of silica sulfuric acid (SSA) as a versatile heterogeneous stable acidic catalyst in organic synthesis has been reported [14-18]. Some advantages of this catalyst are inexpensive, easy to prepare and insoluble in most organic solvents, which could be recycled during work-up.
In view of these observations and also due to our interest in the extension of the Biginelli reaction $[9,10,20,21]$, we wish to report a simple, cheap and convenient method for the synthesis of octahydroquinazolinone using silica sulfuric acid (SSA) as a catalyst.

\section{Experimental}

\subsection{Instrumentation}

All used chemicals were purchased from Merck or Fluka Company. Melting points were determined on an electrothermal digital melting point apparatus. The IR spectra were recorded on Unicom Galaxy series FT-IR 5000 spectrometer. NMR spectra were recorded on a Bruker Avance $(300 \mathrm{MHz})$ spectrometer. Chemical shifts (ppm) were referenced to the internal standard tetramethylsilane (TMS). Microanalyses were performed by the Elemental Analyzer (Elemental, Vario EL III) at the Arak University. The Microanalyses results were agreed favorably with the calculated values. Reactions were monitored by thin layer chromatography using silica gel $\mathrm{F}_{254}$ aluminum sheets (Merck). Silica sulfuric acid (SSA) was prepared according to the method described by literature [14].

\subsection{General procedure for preparation 4a-o}

A solution of benzaldhyde $(2 \mathrm{mmol})$, dimedone $(2 \mathrm{mmol})$ and urea or thiourea $(4 \mathrm{mmol})$ in ethanol $(5 \mathrm{~mL})$ was added silica sulfuric acid $(0.5 \mathrm{~g})$ (Scheme 1). 


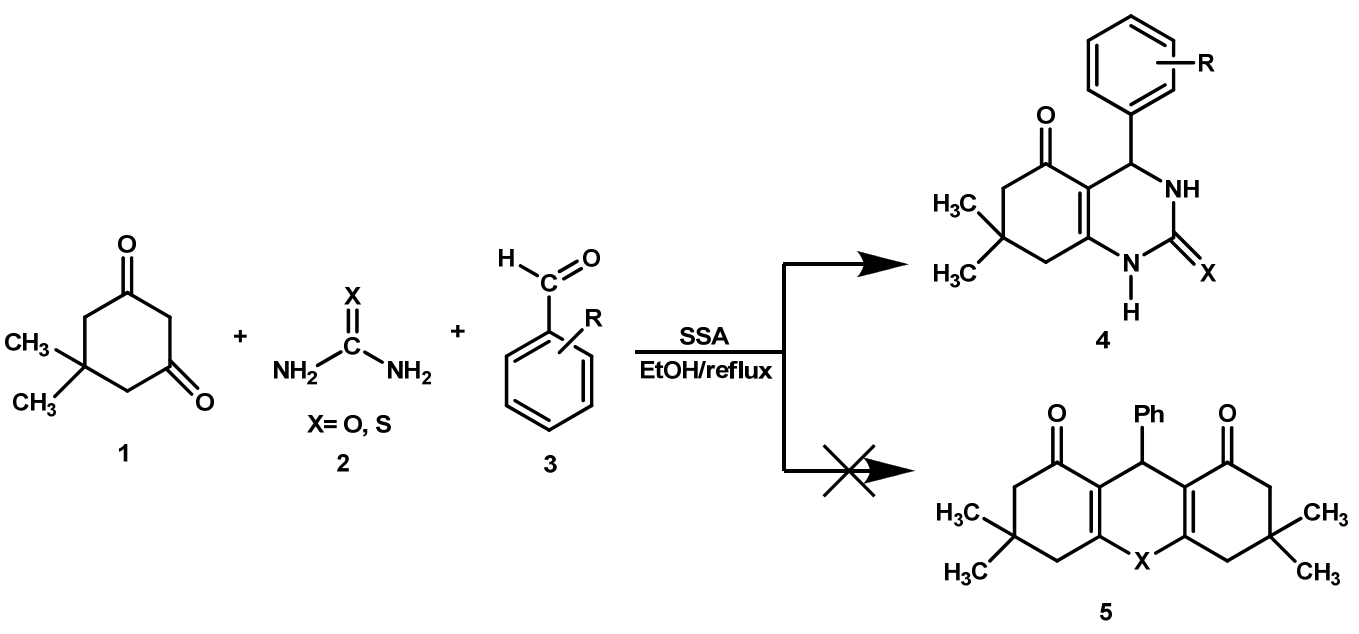

Scheme 1

The reaction mixture was refluxed for desired time and then cooled to room temperature. The solvent was removed and the solid mixture washed with cold water $(50 \mathrm{~mL})$ to remove the excess of urea or thiourea and then filtered. The remaining solid material was washed with hot ethylacetate $(30$ $\mathrm{mL}$ ) and filtered. The filtrate was concentrated, the solid product was separated, air dried, and recrystallized from ethanol to give the pure product.

4-Phenyl-7,7-dimethyl-1,2,3,4,5,6,7,8-octahydroquinazo line-2,5-dione (4a): IR (KBr, $\left.\mathrm{cm}^{-1}\right): 3262,2960,1702,1660$, 1455, 1376. ${ }^{1} \mathrm{H}-\mathrm{NMR}$ (DMSO- $\left.d_{6}, \delta, \mathrm{ppm}\right): 0.89\left(\mathrm{~s}, 3 \mathrm{H}, \mathrm{CH}_{3}\right.$ ), 1.02 (s, 3H, CH3), 2.00-2.44 (m, 4H, $\left.\mathrm{CH}_{2}-8, \mathrm{CH}_{2}-6\right), 5.14$ (d, $J=2.74 \mathrm{~Hz}, 1 \mathrm{H}, H-4), 7.19-7.33(\mathrm{~m}, 5 \mathrm{H}, \mathrm{Ar}-H), 7.78\left(\mathrm{~s}, 1 \mathrm{H}, \mathrm{N}_{3}-\right.$ $\mathrm{H}), 9.48\left(\mathrm{~s}, 1 \mathrm{H}, \mathrm{N}_{1}-\mathrm{H}\right)$. Anal. Calcd. for $\mathrm{C}_{16} \mathrm{H}_{18} \mathrm{~N}_{2} \mathrm{O}_{2}: \mathrm{C}, 71.09 ; \mathrm{H}$, 6.71; N, 10.36\%. Found: C, 71.14; H, 6.62; N 10.24\%.

4-(3,4-Dimethoxy)-7,7-dimethyl-1,2,3,4,5,6,7,8-octahydroqui nazoline-2,5-dione (4c): IR (KBr, cm-1): 3244, 2959, 1701, 1629, 1516, 1376-1451. ${ }^{1} \mathrm{H}-\mathrm{NMR}$ (DMSO- $\left.d_{6}, \delta, \mathrm{ppm}\right): 0.93$ (s, $3 \mathrm{H}$, $\left.\mathrm{CH}_{3}\right), 1.02$ (s, 3H, $\left.\mathrm{CH}_{3}\right), 2.01-2.45$ (m, 4H, $\left.\mathrm{CH}_{2}-8, \mathrm{CH}_{2}-6\right), 3.69$ $\left(\mathrm{s}, 3 \mathrm{H}, \mathrm{OCH}_{3}\right), 3.71\left(\mathrm{~s}, 3 \mathrm{H}, \mathrm{OCH}_{3}\right), 5.09(\mathrm{~d}, J=2.61 \mathrm{~Hz}, 1 \mathrm{H}, H-4)$, 6.70-6.89 (m, 3H, Ar-H), $7.72\left(\mathrm{~s}, 1 \mathrm{H}, \mathrm{N}_{3}-H\right), 9.44\left(\mathrm{~s}, 1 \mathrm{H}, \mathrm{N}_{1}-H\right)$. Anal. Calcd. for $\mathrm{C}_{18} \mathrm{H}_{22} \mathrm{~N}_{2} \mathrm{O}_{4}$ : C, 65.44; $\mathrm{H}, 6.71 ; \mathrm{N}, 8.48 \%$. Found: C, 65.32; H, 6.62; N, 8.54\%.

4-(4-Fluorophenyl)-7,7-dimethyl-1,2,3,4,5,6,7,8-octahydro quinazoline-2,5-dione (4d): IR (KBr, $\left.\mathrm{cm}^{-1}\right)$ : 3239, 2952, 1697, 1631, 1509, 1377-1447. ${ }^{1} \mathrm{H}-\mathrm{NMR}$ (DMSO- $d_{6}, \delta, \mathrm{ppm}$ ): 0.89 (s, $\left.3 \mathrm{H}, \mathrm{CH}_{3}\right), 1.01\left(\mathrm{~s}, 3 \mathrm{H}, \mathrm{CH}_{3}\right), 2.00-2.44\left(\mathrm{~m}, 4 \mathrm{H}, \mathrm{CH}_{2}-8, \mathrm{CH}_{2}-6\right)$, $5.16(\mathrm{~s}, 1 \mathrm{H}, \mathrm{H}-4), 7.10-7.28\left(\mathrm{~m}, 4 \mathrm{H}, \mathrm{H}\right.$ aromatic), $7.75\left(\mathrm{~s}, 1 \mathrm{H}, \mathrm{N}_{3}-\right.$ H), $9.47(\mathrm{~s}, 1 \mathrm{H}, \mathrm{N} 1-\mathrm{H})$. Anal. Calcd. for $\mathrm{C}_{16} \mathrm{H}_{17} \mathrm{FN}_{2} \mathrm{O}_{2}: \mathrm{C}, 66.65 ; \mathrm{H}$, 5.94; N, 9.72\%. Found: C, 66.78; H, 5.91; N, 9.61\%.

4-(4-Nitrophenyl)-7,7-dimethyl-1,2,3,4,5,6,7,8-octahydroquin azoline-2,5-dione (4i): IR (KBr, $\left.\mathrm{cm}^{-1}\right): 3248,2962,1704,1625$, 1523, 1349-1447. ${ }^{1} \mathrm{H}-\mathrm{NMR}$ (DMSO- $d_{6}, \delta, \mathrm{ppm}$ ): 0.87 (s, $3 \mathrm{H}$, $\left.\mathrm{CH}_{3}\right), 1.02\left(\mathrm{~s}, 3 \mathrm{H}, \mathrm{CH}_{3}\right), 2.00-2.46\left(\mathrm{~m}, 4 \mathrm{H}, \mathrm{CH}_{2}-8, \mathrm{CH}_{2}-6\right), 5.30$ (d, J=2.75 Hz, 1H, H-4), 7.51 (d, J=8.76 Hz, 2H, Ar-H), 7.89 (s, $1 \mathrm{H}, \mathrm{N}_{3}-H$ ) 8.20 (d, J=8.78 Hz, 2H, Ar-H), 9.60 (s, 1H, N $1-H$ ). Anal. Calcd. for $\mathrm{C}_{16} \mathrm{H}_{17} \mathrm{~N}_{3} \mathrm{O}_{4}$ : C, 60.95; H, 5.43; N, 13.33\%. Found: C, 61.21; H, 5.51; N, 13.19\%.

4-(4-Methylphenyl)-7,7-dimethyl-5-oxo-1,2,3,4,5,6,7,8-octa hydroquinazoline-2-thione (4k): IR $\left(\mathrm{KBr}, \mathrm{cm}^{-1}\right): 3165,2949$, 1640, 1572, 1475. ${ }^{1} \mathrm{H}-\mathrm{NMR}$ (DMSO- $d_{6}, \delta, \mathrm{ppm}$ ): $0.88(\mathrm{~s}, 3 \mathrm{H}$, $\left.\mathrm{CH}_{3}\right), 1.02\left(\mathrm{~s}, 3 \mathrm{H}, \mathrm{CH}_{3}\right), 2.02-2.47\left(\mathrm{~m}, 7 \mathrm{H}, \mathrm{CH}_{2}-8, \mathrm{CH}_{2}-6, \mathrm{Ar}-\right.$ $\left.\mathrm{CH}_{3}\right), 5.13(\mathrm{~s}, 1 \mathrm{H}, H-4), 7.07-7.14(\mathrm{~m}, 4 \mathrm{H}, \mathrm{Ar}-\mathrm{H}), 9.61\left(\mathrm{~s}, 1 \mathrm{H}, \mathrm{N}_{3}-\right.$ H), $10.51(\mathrm{~s}, 1 \mathrm{H}, \mathrm{N} 1-\mathrm{H})$. Anal. Calcd. for $\mathrm{C}_{17} \mathrm{H}_{20} \mathrm{~N}_{2} \mathrm{OS}$ : C, 67.97; $\mathrm{H}$, 6.71; N, 9.32; \%. Found: C, 68.15; H, 6.49; N, 9.54\%.
4-(3,4-Dimethoxyphenyl)-7,7-dimethyl-5-oxo-1,2,3,4,5,6,7,8octahydroquinazoline-2-thione (41): IR ( $\left.\mathrm{KBr}, \mathrm{cm}^{-1}\right)$ : 3285, 2958, 1627, 1559, 1457. ${ }^{1} \mathrm{H}-\mathrm{NMR}$ (DMSO- $d_{6}, \delta, \mathrm{ppm}$ ): 0.93 (s, $3 \mathrm{H}$, $\left.\mathrm{CH}_{3}\right), 1.03$ (s, 3H, CH $\mathrm{H}_{3}, 2.05-2.42\left(\mathrm{~m}, 4 \mathrm{H}, \mathrm{CH}_{2}-8, \mathrm{CH}_{2}-6\right), 3.70$ $\left(\mathrm{s}, 3 \mathrm{H}, \mathrm{OCH}_{3}\right), 3.71\left(\mathrm{~s}, 3 \mathrm{H}, \mathrm{OCH}_{3}\right), 5.11(\mathrm{~s}, 1 \mathrm{H}, H-4), 6.69-6.92$ $(\mathrm{m}, 3 \mathrm{H}, \mathrm{Ar}-H), 9.65\left(\mathrm{~s}, 1 \mathrm{H}, \mathrm{N}_{3}-H\right), 10.55\left(\mathrm{~s}, 1 \mathrm{H}, \mathrm{N}_{1}-H\right)$. Anal. Calcd. for $\mathrm{C}_{18} \mathrm{H}_{22} \mathrm{~N}_{2} \mathrm{O} 3 \mathrm{~S}$ : C, 68.40; $\mathrm{H}, 6.40 ; \mathrm{N}, 8.09 \%$. Found: C, $68.51 ; \mathrm{H}, 6.32 ; \mathrm{N}, 8.20 \%$.

4-(3-Chlorophenyl)-7,7-dimethyl-5-oxo-1,2,3,4,5,6,7,8octahydroquinazoline-2-thione (4m): $\mathrm{IR}\left(\mathrm{KBr}, \mathrm{cm}^{-1}\right): 3237$, 2954, 1623, 1577, 1459. ${ }^{1} \mathrm{H}-\mathrm{NMR}$ (DMSO- $d_{6}, \delta, \mathrm{ppm}$ ): 0.89 (s, $3 \mathrm{H}, \mathrm{CH} 3), 1.02\left(\mathrm{~s}, 3 \mathrm{H}, \mathrm{CH}_{3}\right), 2.06-2.42\left(\mathrm{~m}, 4 \mathrm{H}, \mathrm{CH}_{2}-8, \mathrm{CH}_{2}-6\right)$, 5.19 (d, J=3.12 Hz, 1H, H-4), 7.16-7.42 (m, 4H, Ar-H), 9.73 (s, $\left.1 \mathrm{H}, \mathrm{N}_{3}-H\right), 10.68\left(\mathrm{~s}, 1 \mathrm{H}, \mathrm{N}_{1}-H\right)$. Anal. Calcd. for $\mathrm{C}_{16} \mathrm{H}_{17} \mathrm{ClN}_{2} \mathrm{OS}$ : C, 59.90; H, 5.34; N, 8.73\%. Found: C, 59.72; H, 5.41; N, 8.75\%.

\section{Results and discussion}

Dihydropyrimidinones, 4a-o were synthesized by reaction of 5,5-dimethyl-1,3-hexadion (dimedone) and corresponding aromatic aldehyde with urea (or thiourea) in the presence of catalytic amount of silica sulfuric acid in refluxing ethanol (Scheme 1). The yield of products was good to excellent without the formation of 1,8-dioxo-octahydoxanthenes $\mathbf{5}$, which is the major product of the procedure reported by the literature [22]. The reaction did not proceed in the absence of SSA after long reaction times. The results are summarized in Table 1. In view of environmentally friendly procedure, the recovery and reuse of this catalyst is quite preferable. Silica sulfuric acid was easily separated from the reaction medium by filtering and regenerated by washing with water and ethanol, followed by drying at room temperature. The catalyst was reused three times for preparation of $\mathbf{4} \mathbf{i}$, as a model reaction, without significant loose of activity (Table 1). Among the various acid catalysts used for preparation of octahydro quinazolinones [9-13,23], the silica sulfuric acid was found more preferable catalyst because of its suitable acidity, availability and low cost.

Yields of this one-pot protocol reactions following recrystallization from ethanol were of the order 76-93\%, which is very favorable as compared with other procedures (46-98\%) $[8,22]$. All obtained products were characterized by NMR and IR spectra as well as their elemental analysis data. In the ${ }^{1} \mathrm{H}-$ NMR spectra of these compounds the two different broad signals at low field were assigned to the resonance of two NHs protons of the pyrimidine ring. This was supported by IR spectra, which included signals in the region $3165-3285 \mathrm{~cm}^{-1}$. 
Table 1. Synthesis of octahydroquinazolinone derivatives using SSA.

\begin{tabular}{|c|c|c|c|c|c|}
\hline Product (4) & $\mathbf{X}$ & $\mathbf{R}$ & Time (h) & Yield (\%)/ (Lit.) & M.P. $\left({ }^{\circ} \mathrm{C}\right) /$ (Lit.) \\
\hline a & 0 & $\mathrm{H}$ & 4 & $90 /(73)[22]$ & $285-286 /(287-290)[22]$ \\
\hline b & 0 & $4-\mathrm{CH}_{3}$ & 3 & $87 /(39)[3]$ & $319 /(>300)[3]$ \\
\hline c & 0 & $3,4-\left(\mathrm{OCH}_{3}\right)_{2}$ & 9 & 82 & $268-270$ \\
\hline d & 0 & $4-\mathrm{F}$ & 5 & 88 & $301-303$ \\
\hline e & 0 & $2-\mathrm{Cl}$ & 8 & $91 /(55)[3]$ & $285 /(282-283)[3]$ \\
\hline f & 0 & $3-\mathrm{Cl}$ & 6 & $93 /(56)[3]$ & $276-278 /(275-276)[3]$ \\
\hline g & 0 & $3-\mathrm{Br}$ & 6 & $85 /(41)[3]$ & $275-277 /(274-275)[3]$ \\
\hline h & 0 & $4-\mathrm{Br}$ & 6 & $90 / 44$ & $330 /(>300)[3]$ \\
\hline i & 0 & $4-\mathrm{NO}_{2}$ & 11 & $80,78,75$ (3 runs) & $303-305$ \\
\hline j & $\mathrm{S}$ & $\mathrm{H}$ & 6 & $85 /(58)[22]$ & $279-281 /(291-293)[22]$ \\
\hline $\mathbf{k}$ & $\mathrm{S}$ & $4-\mathrm{CH}_{3}$ & 4 & $81 /(49)[22]$ & $298-300 /(293-296)[22]$ \\
\hline 1 & $\mathrm{~S}$ & $3,4-\left(\mathrm{OCH}_{3}\right)_{2}$ & 11 & 76 & $230-232$ \\
\hline m & $\mathrm{S}$ & $3-\mathrm{Cl}$ & 8 & 86 & $262-264$ \\
\hline n & $S$ & $3-\mathrm{Br}$ & 8 & 87 & $263-264$ \\
\hline o & $\mathrm{S}$ & $4-\mathrm{Br}$ & 6 & 89 & 284-286 \\
\hline
\end{tabular}

\section{Conclusion}

In conclusion, the present procedure provides an efficient and improved modification of the Biginelli reaction for preparation of octahydroquinazolinone. This procedure is associated with some advantages such as high yields, ease of workup and reusability of catalyst.

\section{Acknowledgement}

We gratefully acknowledge the financial support from the Research Council of the Arak University.

\section{References}

[1]. Kappe, C. O. Eur. J. Med. Chem. 2000, 35, 1043-1052.

[2]. Kung, P. -P.; Casper, M. D.; Cook, K. L.; Wilson-Lingardo, L.; Risen, L. M. Vickers, T. A.; Ranken, R.; Blyn, L. B.; Wyatt, J. R.; Cook, P. D.; Ecker, D. J. J. Med. Chem. 1999, 42, 4705-4713.

[3]. Yarim, M.; Saraç, S.; Kiliç, F. S.; Erol, K. Il Farmaco 2003, 58, 17-24.

[4]. V Alagarsamy, V.; Pathak, U. S.; Sriram, D.; Pandeya, S. N.; Clercq E, De. Ind. J. Pharm. Sci. 2000, 62, 433-437.

[5]. Ladani, N. K.; Patel, M. P.; Patel, R. G, Arkivoc 2009, 7, 292-302.

[6]. Vanden Eynde, J. J.; Godin, J.; Mayence, A.; Maquestiau, A.; Anders, E. Synthesis 1993, 9, 867-869.

[7]. Kempter, G.; Ehrlichmann, W.; Plesse, M.; Lehm, H. -U. J. Pract. Chem. 1982, 324, 832-840.

[8]. Hassani, Z.; Islami, M. R.; Kalantari, M. Bioorg. Med. Chem. Lett. 2000, $16,4479-4482$.

[9]. Mobinikhaledi, A.; Foroughifar, N.; Karimi, G. Synth. React. Inorg. M. 2007, 37, 279-282.

[10]. Zendehdel, M.; Mobinikhaledi. A.; Asgari, A. J. Incl. Phenom. Macro. 2008, 60, 353-357.

[11]. Liu, C. -J.; Wang, J. -D. Molecules 2009, 14, 763-770.

[12]. Dondoni, A.; Massi, A.; Minghini, E.; Sabbatini, S.; Bertolasi, V. J. Org. Chem. 2003, 68, 6172-6183.

[13]. Tajbakhsh, M.; Mohajerani, B.; Heravi, M. M.; Ahmadi, A. N. J. Mol. Catal. A 2005, 236, 216-219.

[14]. Zolfigol, M. A. Tetrahedron 2001, 57, 9509-9511.

[15]. Habibi, D.; Zolfigol, M. A.; Shiri, M.; Sedaghat, A. S. Afr. J. Chem. 2008, 61, 93-96.

[16]. Salehi, P.; Zolfigol, M. A.; Shirini, F.; Baghbanzadeh, M. Curr. Org. Chem. 2006, 10, 2171-2189.

[17]. Habibi, D.; Zolfigol, M. A.; Shirini, F.; Safaiee, M.; Abedini, M.; Rahmani, P. S. Afr. J. Chem. 2007, 60, 17-19.

[18]. Bamoniri, A.; Zolfigoi, M. A.; Mohammadpoor-Baltork, I.; Mirjalili, B. F. J. Ir. Chem. Soc. 2006, 3, 85-88.

[19]. Foroughifar, N.; Mobinikhaledi, A.; Fathinajad, H. Phosphorus. Sulfur. 2003, 178, 1241-1246.

[20]. Mobinikhaledi, A.; Foroughifar, N.; Fathinajad, H. Phosphorus. Sulfur. 2004, 179, 2259-2263.

[21]. Foroughifar, N.; Mobinikhaledi, A.; Shariatzadeh, S. M.; Masoudnia, M. Asian J. Chem. 2002, 14, 782-790.

[22]. Shaabani, A.; Sarvary, A.; Rahmati, A.; Rezayan, A. H. Lett. Org. Chem. 2007, 4, 68-71.

[23]. Sabitha, G.; Reddy, K. B.; Yadav, J. S. Tetrahedron Let. 2003, 44, 6497 6499. 